\title{
In-fiber all-optical modulation based on an enhanced light-matter interaction with graphene
}

\author{
H. Zhang, ${ }^{1, *}$ N. Healy, ${ }^{1,2}$ L. Shen, ${ }^{1}$ C.C. Huang, ${ }^{1}$ D.W. Hewak, ${ }^{1}$ and A.C. Peacock ${ }^{1}$ \\ ${ }^{I}$ Optoelectronics Research Centre, University of Southampton, Highfield, Southampton SO17 1BJ, UK \\ ${ }^{2}$ Emerging Technology and Materials Group, School of Electrical and Electronic Engineering, \\ Newcastle University, Newcastle upon Tyne NE1 7RU, UK \\ hz15e13@soton.ac.uk
}

\begin{abstract}
A graphene-based, high speed, in-fiber optical modulator has been demonstrated on a low-loss side-polished optical fiber platform. These results highlight the potential for robust and efficient integration of low-dimensional materials within standard telecom fibers.

OCIS codes: (060.2310) Fiber optics; (060.2370) Fiber optics sensors; (310.6845) Thin film devices and applications.
\end{abstract}

\section{Introduction}

Graphene is a highly versatile two-dimensional material, exhibiting exceptional optoelectronic properties such as high nonlinearity, high electron mobility, and a strong uniform broadband absorption [1,2]. These properties have been widely explored for the development of a range of broadband photonic devices including polarizers [3], optical limiters [4,5], and wavelength convertors [6].

In this paper, we make use of graphene's strong, ultrafast, saturable absorption to demonstrate a high-speed, infiber optical modulator with a large $9 \mathrm{~dB}$ extinction ratio and an operation bandwidth of $0.5 \mathrm{THz}$. The device is built into a side-polished window of a standard optical fiber, which has an extremely low insertion loss, and is thus immediately compatible with existing fiber networks. Compared to previous reports, our device presents a significant improvement in performance in all key measures to simultaneously obtain low loss, high speed, and high modulation depth. We expect that this significant advancement will open the door to the practical integration of twodimensional materials with traditional optical fibers.

\section{Fabrication and Characterization}

A schematic illustration of our graphene-coated modulator is depicted in Fig. 1(a). The device is developed by applying polyvinyl butyral (PVB)-coated monolayer graphene onto a side-polished standard fiber so that the core guided mode can interact with the graphene film. By retaining a small cladding buffer between the core and the polished surface the insertion loss of the device is less than $1 \mathrm{~dB}$, since the optical path isn't broken. The lightmatter interaction with the graphene film is enhanced via the addition of the high index PVB over-layer, which draws the evanescent tail of the propagating mode out of the core.

To characterize the nonlinear absorption induced by Pauli blocking, we employed a high power fiber laser operating at $1540 \mathrm{~nm}$ with $750 \mathrm{fs}$ duration time and $40 \mathrm{MHz}$ repetition rate. Fig. 1(b) shows the transmittance of the TE and TM modes as a function of increasing average power coupled into the device. From this result we see that the absorption of the TM mode is linear up to $10 \mathrm{~mW}$ input power, while for higher input powers the absorption becomes nonlinear and the transmitted light starts to increase exponentially, eventually reaching a transmittance of $50 \%$ for $32 \mathrm{~mW}$ input power. In contrast, the transmission of the TE mode remains largely unchanged when the input power is increased up to the maximum available power, evidence of the minimal surface wave coupling for this orientation of the polarization.

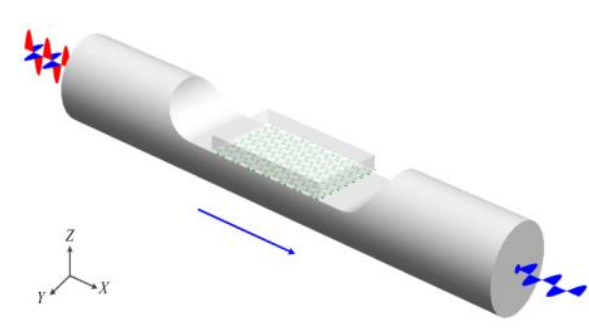

(a)

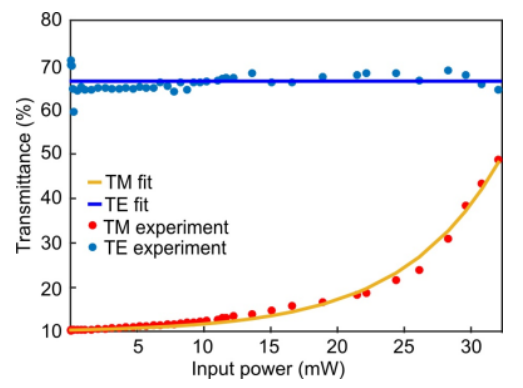

(b)

Fig. 1. (a) Schematic illustration of the modulator. (b) Transmittance measurements as a function of input power. 


\section{Results and Discussion}

In order to determine the temporal dynamics of the saturable absorption, a simple pump-probe modulation scheme was employed, as illustrated in Fig. 2(a). In this set-up, the $1540 \mathrm{~nm}$ fiber laser was split into a high power pump (20 $\mathrm{mW}$ average power, corresponding to a peak power of $720 \mathrm{~W})$ and a weak probe component $\left(\mathrm{P}_{\text {ave }}=130 \mu \mathrm{W}, \mathrm{P}_{\mathrm{p}}=4\right.$ W). The probe was modulated at $100 \mathrm{~Hz}$ using an optical chopper connected to a lock-in amplifier to discriminate between the two signals. Polarization controllers were used to ensure that both the pump and probe were aligned to couple into the TM surface wave, and a 10 ps adjustable delay was inserted into the probe's path to control the overlap between the two pulses. The pump and probe pulses were then recombined using a fused tapered coupler before launching into the device under test. Fig. 2(b) shows that the power of the transmitted probe signal can be modulated with an extinction ratio of $\sim 9 \mathrm{~dB}$, over a timescale of $2 \mathrm{ps}$ as the pump-probe overlap is tuned. Overlaying the measured response with that of the pump itself, it is clear that the rise time is simply governed by the pump duration, however the fall time is slightly longer, which can be attributed to the intrinsic carrier recovery processes in graphene. These relaxation processes occur on two different timescales, which we estimate from the response to be $\tau_{1} \sim 0.1 \mathrm{ps}$ and $\tau_{2} \sim 0.4 \mathrm{ps}$, in good agreement with previous reports [7].

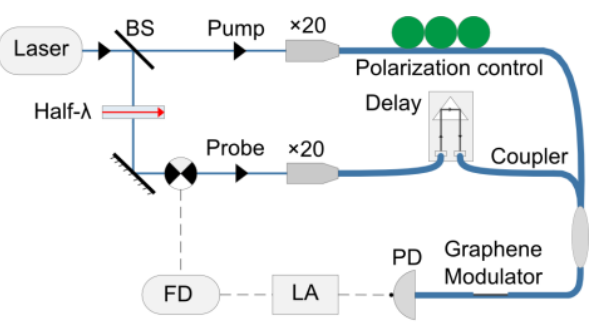

(a)

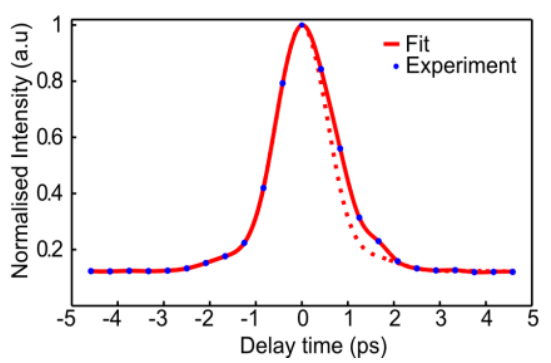

(b)

Fig. 2. (a) A schematic illustration of the experimental set-up used for high intensity optical characterization. BS: beam splitter; FD: frequency driver; LA: lock-in amplifier; PD: photodiode. (b) Temporally resolved speed of the all-optical modulator. The dashed red line is mirroring of the rising edge of the pulse.

\section{Conclusion}

We have designed and experimentally demonstrated a graphene-based all-optical modulator with a high extinction ratio of $9 \mathrm{~dB}$ and a speed of $0.5 \mathrm{THz}$. When compared to previous devices, our method results in an enhanced interaction with the graphene sheet and a corresponding significant increase in modulation depth, while at the same time ensuring a large decrease in insertion loss. The side-polished fiber device is robust and stable, and it can be packaged using methods that are well-established for existing fiber devices, enabling immediate compatibility with standard infrastructures. We expect that this method for enhancing light-matter interactions could be easily transferred to other two-dimensional materials to realize functionalized side-polished fiber devices that are capable of generating, modulating, and detecting light in next generation all-fiber optoelectronic systems.

\section{References}

[1] E. Hendry, P. J. Hale, J. Moger, A. K. Savchenko, and S. A. Mikhailov, "Coherent nonlinear optical response of graphene,” Phys. Rev. Lett. 105, 097401 (2010).

[2] R. Nair, P. Blake, A. Grigorenko, K. Novoselov, T. Booth, T. Stauber, N. Peres, and A. Geim, "Fine structure constant defines visual transparency of graphene," Science 320, 1308-1308 (2008).

[3] Q. Bao, H. Zhang, B. Wang, Z. Ni, C. H. Y. X. Lim, Y. Wang, D. Y. Tang, and K. P. Loh, "Broadband graphene polarizer," Nat. Photon. 5 411-415 (2011).

[4] M. Feng, H. Zhan, and Y. Chen, "Nonlinear optical and optical limiting properties of graphene families," Appl. Phys. Lett. 96, 033107 (2010).

[5] Z. Sun, D. Popa, T. Hasan, F. Torrisi, F. Wang, E. J. Kelleher, J. C. Travers, V. Nicolosi, and A. C. Ferrari, "A stable, wideband tunable, near transform-limited, graphene-mode-locked, ultrafast laser," Nano Res. 3, 653-660 (2010).

[6] Y. Wu, B. C. Yao, Q. Y. Feng, X. L. Cao, X. Y. Zhou, Y. J. Rao, Y. Gong et al., "Generation of cascaded four-wave-mixing with graphenecoated microfiber," Photon. Res. 3, A64-A68 (2015).

[7] J. M. Dawlaty, S. Shivaraman, M. Chandrashekhar, F. Rana, and M. G. Spencer, "Measurement of ultrafast carrier dynamics in epitaxial graphene,” Appl. Phys. Lett. 92, 042116 (2008). 